\title{
Comparison of treatment outcomes in chronic coccygodynia patients treated with ganglion impar blockade versus caudal epidural steroid injection: a prospective randomized comparison study
}

\author{
Savas Sencan ${ }^{1}$, Gunay Yolcu ${ }^{2}$, Serhad Bilim ${ }^{3}$, Ozge Kenis-Coskun $^{2}$, and Osman Hakan Gunduz ${ }^{1}$ \\ 'Division of Pain Medicine, Department of Physical Medicine and Rehabilitation, Faculty of Medicine, Marmara University, Istanbul, Turkey \\ ${ }^{2}$ Department of Physical Medicine and Rehabilitation, Faculty of Medicine, Marmara University, Istanbul, Turkey \\ ${ }^{3}$ Pain Clinic, Adryaman Education and Research Hospital, Adlyaman, Turkey
}

Received August 4, 2021

Revised October 5, 2021

Accepted October 6, 2021

Handling Editor: Younghoon Jeon

Correspondence

Gunay Yolcu

Department of Physical Medicine and Rehabilitation, Marmara University Medical School Pendik Research and Training Hospital, Fevzi Çakmak Mahallesi, Tepe Sokak, No: 41, Üst Kaynarca, Pendik, Istanbul 34899, Turkey

Tel: +90 2166570606

Fax: +90 2166254639

E-mail: dryolcugunay@gmail.com
Background: Coccygodynia is one of the chronic, refractory painful musculoskeletal disorders. Interventional procedures are applied to patients unresponsive to initial treatment in coccygodynia. This study aims to compare the treatment outcomes of ganglion impar block (GIB) and caudal epidural steroid injection (CESI) in patients with chronic coccygodynia.

Methods: This study was a prospective randomized comparison study conducted between June 2019 and January 2021. Patients diagnosed with chronic coccygodynia were randomly divided into two groups: the GIB group and the CESI group. The severity of pain, presence of neuropathic pain, and quality of life were evaluated using the Numeric Rating Scale, Leeds Assessment of the Neuropathic Symptoms and Signs Scale, and Short Form-12 Health Survey (SF-12), respectively.

Results: A total of 34 patients in each group were included in the final analyses. While there was a significant decrease in pain intensity in both groups in the 3-month follow-up, this decrease was more significant in the GIB group at the 3rd week. There was a significant improvement in the SF-12 physical score and the number of patients with neuropathic pain in both groups in the 3rd week, but this improvement was not observed in the 3rd month.

Conclusions: Although GIB may provide more pain relief in short term, both GIB and CESI are useful treatment methods in coccygodynia unresponsive to more conservative treatments.

Key Words: Chronic Pain; Coccyx; Ganglia, Sympathetic; Injections, Epidural; Musculoskeletal Pain; Neuralgia; Pain Measurement; Pelvic Girdle Pain; Quality of Life; Steroids; Treatment Outcome.

\section{INTRODUCTION}

Coccygodynia (or coccydynia) is defined as the painful and disabling condition of the coccyx (tailbone) located inferior to the sacrum. Although it was known as early as the 18th century, there are still uncertainties in terms of multifactorial pain pathophysiology and effective treatment modalities [1]. (a) This is an open-access article distributed under the terms of the Creative Commons Attribution Non-Commercial License (http://creativecommons.org/licenses/by-nc/4.0/), which permits unrestricted non-commercial use, distribution, and reproduction in any medium, provided the original work is properly cited.

(C) The Korean Pain Society, 2022
Author contributions: Savas Sencan: Study conception; Gunay Yolcu: Writing/manuscript preparation; Serhad Bilim: Data curation; Ozge Kenis Coskun: Writing/manuscript preparation; Osman Hakan Gunduz: Supervision. 
Most patients have experienced a prior traumatic event such as a fall on the tailbone, childbirth, or lumbar spine surgery, whereas some cases are idiopathic [2]. Prolonged sitting, defecation, standing up from a sitting position, and leaning back while sitting may worsen the pain [3]. The prevalence of coccygodynia is five times more in females than in males, associated with the fact that the coccyx is more prone to trauma because of childbirth and its more posterior location in females [4]. Initial treatment is typically rest, use of pressure-relieving cushions, leaning forward while sitting, hot or ice pack application based on patients' preference, as well as oral analgesics such as nonsteroidal anti-inflammatory drugs or paracetamol [5].

Interventional procedures are applied to patients whose pain is not relieved by initial treatment. Ganglion impar block (GIB) under fluoroscopic guidance has recently been frequently used in the treatment of chronic coccygodynia $[6,7]$. The ganglion impar is the sympathetic ganglion located anterior to the coccyx. The block of this ganglion with a steroid and local anesthetic has been shown to relieve both the somatic and neuropathic pain components of chronic coccygodynia [8]. Another treatment option that is relatively less emphasized in the literature, but frequently used in clinical practice, is the caudal epidural steroid injection (CESI) [9]. Although the mechanism of action has not been determined precisely, limited data on its effectiveness support CESI $[3,10]$.

To our best knowledge, there is no prospectively designed study comparing these two interventional methods in the literature. The aim of this study is to compare the treatment outcomes of GIB and CESI in patients with chronic coccygodynia unresponsive to conservative treatment.

\section{MATERIALS AND METHODS}

This study was a prospective randomized comparison study conducted between June 2019 and January 2021 (clinical trial registration number: NCT04902742). Ethics committee approval was obtained from the Committee of Ethics of Marmara University (Ethics number: 09.2019.395). Patients who have suffered from coccygeal pain for at least 3 months and been diagnosed with chronic coccygodynia in our pain clinic in the subsequent stage, with a clinical and physical examination, as well as radiologic assessments, were included in the study. Other inclusion criteria were intractable pain over the coccyx despite conservative treatment and being between the ages of 18 and 65. Patients with a history of GIB and/or CESI carried out in the previous 3 months, patients with a history of lumbar surgery, patients with systemic and/or local infections, malignancy, bleeding diathesis, acute fracture, known allergy to contrast material and/or local anesthetic substances, known history of any psychiatric disorder, and patients with a history of pregnancy were excluded from the study. Verbal and written informed consent were obtained from all patients participating in the study.

After the enrollment period, all patients were loaded into the computer program for the randomization process and randomly divided into two groups: a GIB group and a CESI group. The patients did not know which treatment group they were in, however, the clinician who performed the interventional procedure knew which method he was using. In this way, our study was conducted with a single blind design. It was also ensured that the clinician who evaluated the patients during the follow-up periods did not know which group the patients were in, thus assessor blinding was provided.

\section{Procedure techniques}

GIB was conducted with the aid of a fluoroscope by a pain medicine expert with at least 10 years of experience in this field. The patients were made to lie down in a prone position, and their intergluteal regions were made aseptic. After the sacrococcygeal joint was visualized with the lateral fluoroscopic view, local anesthesia was applied at the sacrococcygeal junction via $3 \mathrm{~mL} 2 \%$ prilocaine, and the infiltration was applied to both cutaneous and subcutaneous tissues. The sacrococcygeal joint was pierced with a 22-gauge spinal needle, and the ganglion impar was reached. After a $1 \mathrm{~mL}$ injection of non-ionic contrast medium, there was dye dispersion without vascular spread, and an image of an "inverse comma" sign was detected in the lateral visualization (Fig. 1A); $3 \mathrm{~mL} 0.5 \%$ bupivacaine, $1 \mathrm{~mL}$ of saline, and $40 \mathrm{mg}$ of methylprednisolone acetate were injected into the patients.

For CESI, the patients were placed in a prone position, and the caudal epidural space was visualized with fluoroscopic guidance. After subcutaneous local anesthetic infiltration at the injection site, an 18-gauge Touhy needle was advanced into the caudal epidural space with intermittent fluoroscopy imaging. After determining negative blood aspiration and cerebrospinal fluid, the epidural space was confirmed in the lateral and anteroposterior views with 1 $\mathrm{mL}$ of non-ionic radiopaque material. A mixture of $2 \mathrm{~mL}$ $0.5 \%$ bupivacaine, $1 \mathrm{~mL}$ of saline, and $80 \mathrm{mg}$ of triamcinolone acetonide was injected into the caudal epidural space (Fig. 1B).

The patients were observed for 2 hours post-procedure in the recovery room to monitor for any complications. 


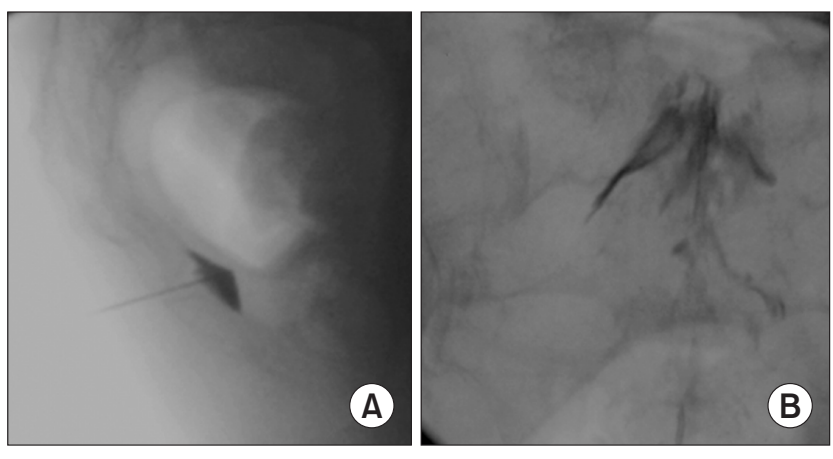

Fig. 1. (A) Image of an "inverse comma" sign at ganglion impar block. (B) Spread of the mixture in the caudal epidural space at caudal epidural steroid injection.

\section{Assessment scales}

In addition to the recording of the demographic data of the patients participating in this study (age, sex, duration of symptoms, etc.), the numeric rating scale (NRS) was employed before the procedure and in the 1st hour, 3rd week, and 3rd month after the procedure; the Leeds Assessment of Neuropathic Symptoms and Signs Scale (LANSS) and Short Form-12 Health Survey (SF-12) were employed before the procedure and in the 3rd week and 3rd month after the procedure.

The NRS is a frequently used method in measuring the severity of the pain and in monitoring it. It is an 11-point scale, in which 0 means no pain and 10 means the most severe pain possible, and the patient is asked to score his/her pain between 0 and 10. LANSS was developed to evaluate the neuropathic component of pain. It has one part of pain questions and a part to evaluate touch and pinprick sensation. On the LANSS Pain Scale, a score of 12 or more was classified as neuropathic pain, and a score under 12 was classified as nociceptive pain. It does not reflect the severity of pain or neuropathic pain itself, just the presence and absence of it. Quality of life has been evaluated by SF12 , which is a health-related quality-of-life questionnaire consisting of twelve questions that measure eight health domains to assess physical and mental health. The LANSS and SF-12 scales are easily administered and validated in Turkish [11,12].

In this study, the researcher conducting all the interviews had no knowledge of which groups each patient belonged to.

\section{Statistical analyses}

Sample sizing was performed using $\mathrm{G}^{*}$ power version 3.1 (Heinrich-Heine-Universität Düsseldorf, Düsseldorf, Germany). According to a similarly designed previous study, the required patient number was calculated as at least 30 patients in each group for an $\alpha$ of 0.05 and a power of 0.80 , with an anticipated $15 \%$ change in the NRS scores [13]. SPSS version 22.0 (IBM Corp., Armonk, NY) was used in statistical analyses. There were no missing data. Basic methods were used for descriptive analyses. For demographic properties and the initial differences between the two groups, crosstabs and independent samples $t$-tests were used. For the presence and absence of neuropathic pain between and within groups, the Fischer's exact and McNemar tests were used. The distribution of other data was assessed using the Shapiro-Wilk test and Q-Q plots. The data were distributed normally. For the assessment of the effects within each group, repeated-measures analysis of variance (ANOVA) with a Bonferroni correction was used. A $P$ value below 0.017 was accepted as significant. For group differences, two-way mixed ANOVA was used. A $P$ value below 0.05 was accepted as significant.

\section{RESULTS}

A total of 73 chronic coccygodynia patients accepted enrollment in the study. Thirty-seven patients were allocated to the GIB group and 36 patients to the CESI group. Five patients were lost during follow-up periods, 3 patients from the GIB group and 2 patients from the CESI group. A total of 34 patients in each group were included in the final analyses (Fig. 2).

Patients' demographic properties and initial assessments were given in Table 1. There were no significant differences between the two groups in any of the initial parameters. In both groups, all patients had significant pain relief after injection, all showing a decrease of $80 \% 1$ hour after injection. Mean NRS in 1st hour was $1.0 \pm 1.6$ in the CESI group and $0.7 \pm 1.4$ in the GIB group and were not significantly different $(P=0.413)$. When each group was analyzed within itself, both groups showed significant improvements in pain at each checkpoint. Pain scores in the GIB group were significantly better in the 3rd week, but there were no significant differences in the 3rd month. SF12 physical scores improved at the 3rd week mark in both groups, however this improvement was not seen in the 3rd month. No improvement in SF-12 mental scores was seen in either group. There were no significant differences between the quality-of-life scores at all checkpoints between groups. All findings are summarized in Table 2 and Fig. 3.

When the groups were analyzed for the presence of neuropathic pain, the data demonstrated that both groups also showed significant improvements. The number of patients with neuropathic pain was 16 before the injection and 8 at the $3 r d$ week in the CESI group $(P=0.012)$. These 


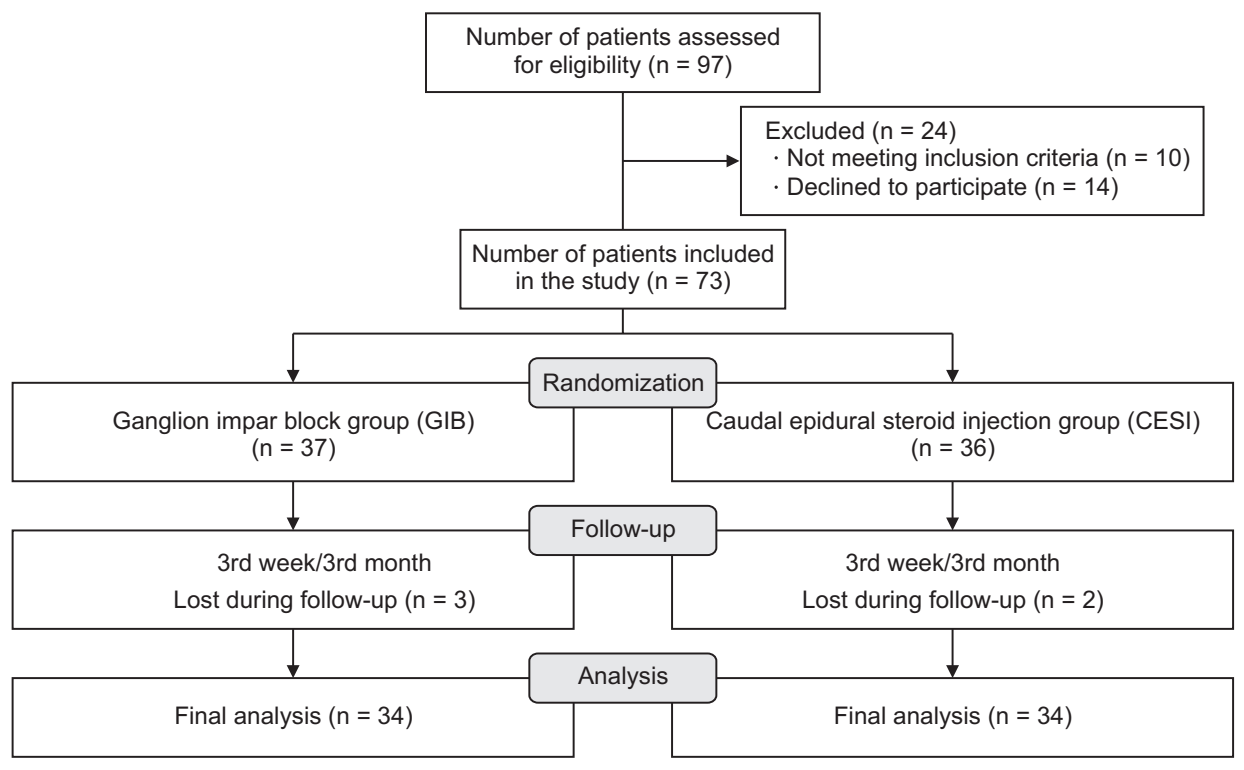

Fig. 2. Flow diagram.

Table 1. Demographic properties and initial assessments of the patients

\begin{tabular}{|c|c|c|c|}
\hline Variable & CESI group $(n=34)$ & GIB group $(n=34)$ & $P$ value \\
\hline Age (yr) & $46.2 \pm 11.9$ & $46.0 \pm 13.7$ & 0.953 \\
\hline Female & $26(76.5)$ & $23(67.6)$ & 0.412 \\
\hline $\mathrm{BMI}\left(\mathrm{kg} / \mathrm{m}^{2}\right)$ & $26.7 \pm 4.5$ & $27.7 \pm 4.6$ & 0.364 \\
\hline Symptom duration (mo) & $12.4 \pm 7.8$ & $11.9 \pm 6.3$ & 0.981 \\
\hline Positive history of trauma & $20(58.8)$ & $19(55.9)$ & 0.800 \\
\hline Initial NRS & $8.5 \pm 1.0$ & $7.9 \pm 1.2$ & 0.200 \\
\hline Initial SF-12 & $40.4 \pm 13.3$ & $39.3 \pm 13.6$ & 0.724 \\
\hline Presence of neuropathic pain according to LANSS & $18(52.9)$ & $18(52.9)$ & $>0.999$ \\
\hline \multicolumn{4}{|l|}{ Drugs } \\
\hline NSAIDs & $13(38.2)$ & $11(32.4)$ & 0.724 \\
\hline Paracetamol & $4(11.8)$ & $4(11.8)$ & $>0.999$ \\
\hline Tramadol & $3(8.8)$ & $2(5.9)$ & 0.663 \\
\hline Gabapentinoids & $14(41.2)$ & $14(41.2)$ & $>0.999$ \\
\hline Duloxetine & $6(17.6)$ & 5 (14.7) & 0.762 \\
\hline
\end{tabular}

Values are presented as mean \pm standard deviation or number (\%).

CESI: caudal epidural steroid injection, GIB: ganglion impar block, BMI: body mass index, NRS: numeric rating scale, SF-12: Short Form-12 Health Survey, LANSS: Leeds Assessment of Neuropathic Symptoms and Signs Scale, NSAID: nonsteroidal anti-inflammatory drug.

values were 16 before the injection and 10 at the 3 rd week for the GIB group $(P=0.014)$. However, in the 3rd month, the GIB group had 15 patients with neuropathic pain, while this number was 14 for the CESI group, and was not significantly different from the initial assessment $(P=0.684$ for both analyses). There were no significant differences in each checkpoint between the groups $(P=0.204 ; P=0.343)$.

After the interventions, two patients in the GIB group developed hypotension due to vasovagal reactions. After the short-term follow-up, both patients, whose symptoms were relieved and findings improved, were discharged with recommendations. No other side effects or complications were encountered.

\section{DISCUSSION}

In this study, both GIB and CESI were shown to cause pain reduction in coccygodynia. It was also shown that both methods might have short-term beneficial effects on the neuropathic pain component. These results are a crucial contribution to the literature comparing the effectiveness of both interventional treatment methods prospectively for the first time.

As a sympathetic ganglion, the ganglion impar is the endpoint of the paravertebral sympathetic chain, and subtends the nociception and sympathetic innervation of the perineal region [14]. The efficacy of the GIB as a popular treatment option has been previously shown in many studies $[6,15,16]$. The GIB can be performed with fluoros- 


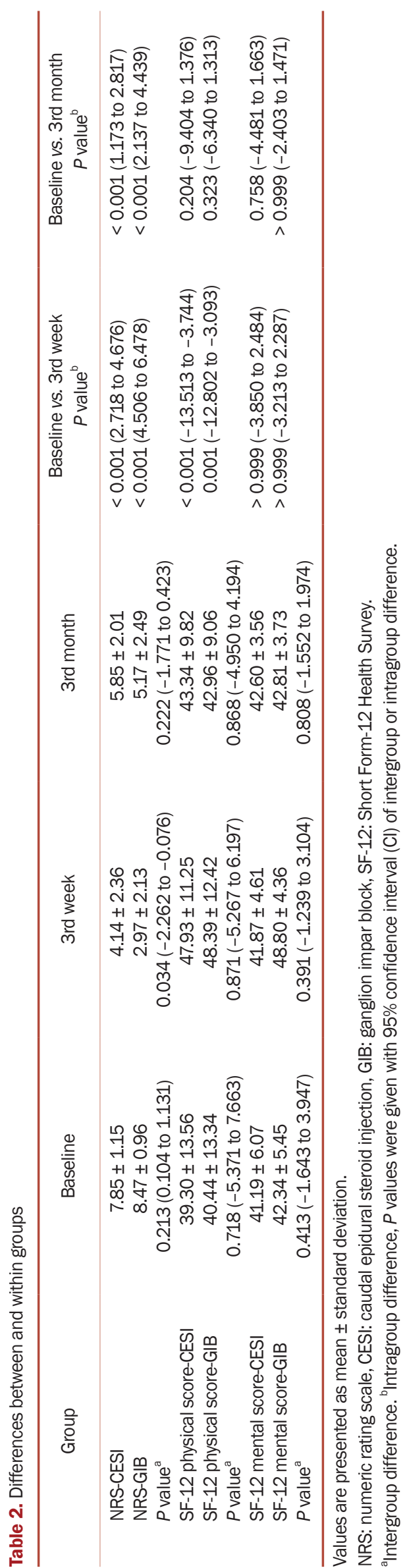

copy, computerized tomography, or ultrasound guidance with no well-defined superiority [17]. In the blockage, a mixture of local anesthetic and steroids can be used, as well as an only local anesthetic. It was preferred to add steroids, as the addition of steroids appeared to produce a greater reduction in pain in a previous comparative study [13]. In this study, it was found that the GIB provided a more significant pain reduction in the 3rd week. The ganglion impar is a relay point that has an important role in sympathetically mediated pain transmission and neuropathic pain pathophysiology. As a more definitive target, GIB may be more beneficial in pain reduction than CESI, which acts as a peripheral mechanism through the sacrococcygeal nerves. Although GIB generally provides an adequate clinical response in patients with coccygodynia from to non-malignant causes, ganglion impar neurolysis or radiofrequency ablation procedure can be applied in patients with persistant pain mostly seen in pelvic and perineal cancers [18]. Neurolytic blocks consist of the destruction of nervous tissue usually through the injection of alcohol or phenol.

CESI is a frequently used treatment method in disorders causing low back pain. Although it is frequently used in clinical practice and stated as a method that can be applied in patients who do not respond to conservative treatment $[10,19]$, there is a shortage of high-quality studies on coccydynia. In a recently published retrospective study, the efficacy of the GIB and CESI combined with transrectal manipulation was compared, and the former was found to be more effective in improving pain sensation and painless sitting periods in a 6-month follow-up [10]. One of the striking results of this study was the limited reduction in pain in the CESI with manipulation group. The effect of CESI on pain reduction can be explained by the relief of pain associated with lumbar pathology and blockage of the sacrococcygeal nerves, which is an important source of pain in coccydynia. There is a close relationship between coccydynia and lumbar pathologies such as disc herniation, disc degeneration, and spinal stenosis as an important target of CESI $[1,20]$. Pulsed radiofrequency and neuromodulation interventions can also be performed via the caudal epidural route for the sacrococcygeal nerves as a peripheral target [21,22]. Further studies are still needed to understand the effectiveness of CESI and other interventions with a caudal approach in coccygodynia more comprehensively.

Another point to be emphasized about CESI is that there is no consensus on the optimal injectable volume for coccygodynia. In lumbar pathologies, a high volume is preferred due to the distance to the targeted area, but it is thought that clinical benefit can be obtained with a lower volume due to the closeness of the sacral roots in coccygo- 

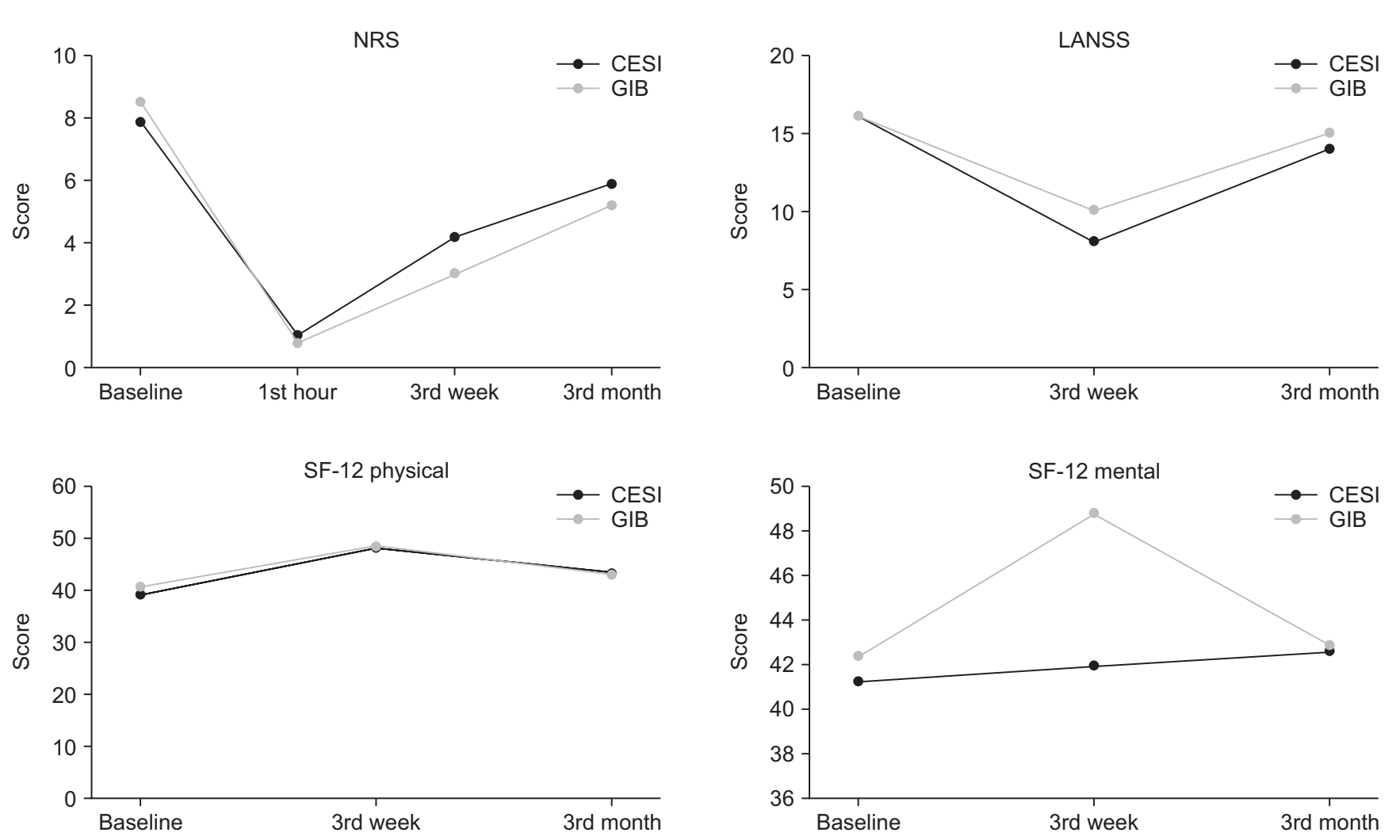

Fig. 3. Change in main outcome measures in follow-up periods (mean scores were given for NRS and SF-12, while numbers of patients with neuropathic pain were shown for LANSS). CESI: caudal epidural steroid injection, GIB: ganglion impar block, NRS: numeric rating scale, LANSS: Leeds Assessment of Neuropathic Symptoms and Signs Scale, SF-12: Short Form-12 Health Survey.

dynia. In addition, it has been reported that complications related to compression such as anosmia and hiccup can be seen due to volume effect in CESI $[23,24]$. We preferred to use a lower injectable volume, but further studies are needed to examine the effect of volume on clinical outcomes of CESI in patients with coccygodynia. Chronic coccygeal pain can have a neuropathic component that should also be evaluated in order to provide effective pain reduction. It has been previously shown that GIB is effective in decreasing the neuropathic component of pain in chronic coccygodynia with a 6 -month follow-up period [8]. In this study, while improvement was observed in both groups in the 3rd week, it was found that this improvement did not persist in the 3rd month. To explain these differences in neuropathic pain outcomes, it must also be kept in mind that the LANSS reflects the presence or absence of neuropathic pain without assessing severity. More objective assessments are needed to better understand all aspects of pain mechanisms. With the possible negative contribution of the neuropathic component of pain, assessment of the quality of life has crucial importance on coccydynia. In this study, the SF-12 physical scores were found to be improved at the 3rd week in both groups. This finding can be explained by a reduction in pain and providing pain-free sitting. However, a recent study found that the SF-12 physical score improved significantly in the 3rd month without associated pain-free sitting, while this improvement was not seen in the 6th month [8]. The point to consider when evaluating differences in results is that existing quality of life measurements are not explicitly made for coccygodynia.

Since there are no well-defined guidelines on the treatment of coccygodynia, the treatment options should be decided considering the positive and negative aspects on an individualized basis. It has been reported that some rare complications such as rectal rupture, neuritis, and cauda equina syndrome may be seen [25], although these decrease under the guidance of imaging. The GIB provides greater pain reduction. However, the risk of disc-related infection (discitis) should also be considered. CESI may be preferred in patients with accompanying low back pain under appropriate conditions. It is important to evaluate clinical and pathophysiological factors that cause coccygeal pain in each patient to determine the most beneficial treatment method.

This study has some limitations. Due to the short followup time, it is unclear that efficacy continues for both groups over the long term and whether repeated injections would be required. Different results may have been found in the CESI group, as possible pathologies that may cause 
low back pain had not been evaluated. Functionality could not be evaluated because of the absence of specific scales directed at chronic coccygodynia. Finally, the study did not include a placebo control group for ethical reasons. Nonetheless, because this is the first comparative, prospective study regarding GIB and CESI, the results of this study should arouse interest in more comprehensive studies.

In conclusion, both the GIB and CESI are useful treatment methods in coccygodynia unresponsive to more conservative treatments. The GIB may provide more significant pain reduction in the short term. Further studies with a long follow-up period and more objective assessments for the neuropathic component of pain are warranted to understand the efficacy of both treatment methods more precisely.

\section{CONFLICT OF INTEREST}

No potential conflict of interest relevant to this article was reported.

\section{FUNDING}

No funding to declare.

\section{ORCID}

Savas Sencan, https://orcid.org/0000-0001-8150-0581

Gunay Yolcu, https://orcid.org/0000-0002-8545-7821

Serhad Bilim, https://orcid.org/0000-0001-7101-4977

Ozge Kenis-Coskun, https://orcid.org/0000-0002-3004-1992

Osman Hakan Gunduz, https://orcid.org/0000-0002-3214-803X

\section{REFERENCES}

1. Garg B, Ahuja K. Coccydynia-a comprehensive review on etiology, radiological features and management options. J Clin Orthop Trauma 2021; 12: 123-9.

2. Patijn J, Janssen M, Hayek S, Mekhail N, Van Zundert J, van Kleef M. 14. Coccygodynia. Pain Pract 2010; 10: 554-9.

3. Lirette LS, Chaiban G, Tolba R, Eissa H. Coccydynia: an overview of the anatomy, etiology, and treatment of coccyx pain. Ochsner J 2014; 14: 84-7.

4. Karadimas EJ, Trypsiannis G, Giannoudis PV. Surgical treatment of coccygodynia: an analytic review of the literature. Eur Spine J 2011; 20: 698-705.

5. Foye PM. Coccydynia: tailbone pain. Phys Med Rehabil Clin
N Am 2017; 28: 539-49.

6. Gunduz OH, Sencan S, Kenis-Coskun O. Pain relief due to transsacrococcygeal ganglion impar block in chronic coccygodynia: a pilot study. Pain Med 2015; 16: 1278-81.

7. Gonnade N, Mehta N, Khera PS, Kumar D, Rajagopal R, Sharma PK. Ganglion impar block in patients with chronic coccydynia. Indian J Radiol Imaging 2017; 27: 324-8.

8. Sencan S, Kenis-Coskun O, Demir FGU, Cuce I, Ercalık T, Gunduz OH. Ganglion Impar block improves neuropathic pain in coccygodynia: a preliminary report. Neurol Neurochir Pol 2018; 52: 612-7.

9. Elkhashab Y, Ng A. A review of current treatment options for coccygodynia. Curr Pain Headache Rep 2018; 22: 28.

10. Govardhani Y, RamMohan G, Abhijith S, Savithri B. A comparative retrospective study of the efficacy of caudal epidural with manipulation versus ganglion impar block with manipulation in patients with coccydynia. Indian J Pain 2021; 35: 42-5.

11. Yucel A, Senocak M, Kocasoy Orhan E, Cimen A, Ertas M. Results of the Leeds assessment of neuropathic symptoms and signs pain scale in Turkey: a validation study. J Pain 2004; 5: 427-32.

12. Soylu C, Kütük B. Reliability and validity of the Turkish version of SF-12 Health Survey. Turk Psikiyatri Derg 2021 [in press]

13. Sencan S, Edipoglu IS, Ulku Demir FG, Yolcu G, Gunduz $\mathrm{OH}$. Are steroids required in the treatment of ganglion impar blockade in chronic coccydynia? A prospective doubleblinded clinical trial. Korean J Pain 2019; 32: 301-6.

14. Oh CS, Chung IH, Ji HJ, Yoon DM. Clinical implications of topographic anatomy on the ganglion impar. Anesthesiology 2004; 101: 249-50.

15. Sagir O, Demir HF, Ugun F, Atik B. Retrospective evaluation of pain in patients with coccydynia who underwent impar ganglion block. BMC Anesthesiol 2020; 20: 110.

16. Sir E, Eksert S. Comparison of block and pulsed radiofrequency of the ganglion impar in coccygodynia. Turk J Med Sci 2019; 49: 1555-9.

17. Ghai A, Jangra P, Wadhera S, Kad N, Karwasra RK, Sahu A, et al. A prospective study to evaluate the efficacy of ultrasoundguided ganglion impar block in patients with chronic perineal pain. Saudi J Anaesth 2019; 13: 126-30.

18. Ferreira F, Pedro A. Ganglion impar neurolysis in the management of pelvic and perineal cancer-related pain. Case Rep Oncol 2020; 13: 29-34.

19. Foye PM, Buttaci CJ, Stitik TP, Yonclas PP. Successful injection for coccyx pain. Am J Phys Med Rehabil 2006; 85: 783-4.

20. De Andrés J, Chaves S. Coccygodynia: a proposal for an algorithm for treatment. J Pain 2003; 4: 257-66.

21. Atim A, Ergin A, Bilgiç S, Deniz S, Kurt E. Pulsed radiofrequency in the treatment of coccygodynia. Agri 2011; 23: 1-6.

22. Lee DW, Lai A. Sacral burst neuromodulation via caudal ap- 
proach as a treatment for chronic coccydynia. Neuromodulation 2019; 22: 992-4.

23. Kaydu A, Kiliç ET, Gökçek E, Kaçar CK. Anosmia after caudal epidural steroid injection. Anesth Essays Res 2018; 12: 291-3.

24. Kaydu A, Kılıç ET, Gökçek E, Akdemir MS. Unexpected com- plication after caudal epidural steroid injection: hiccup. Anesth Essays Res 2017; 11: 776-7.

25. Gupta N, Garg R, Saini S, Bharti SJ, Kumar V. An unusual complication after ganglion impar block for chronic cancer pain management. AANA J 2017; 85: 424-6. 